\title{
Methods of Early Diagnostics Fiber Optical Communication Lines
}

\author{
Nushtaeva Ozoda Xasilovna, Alimjonov Botirjon Abdulmaqsud o'g'li, Yusupova Muhtabar \\ Muminjanovna
}

\begin{abstract}
This article investigates major methods of early diagnostics fiber optical communication lines. On this way, research points of the optical communication were analyzed in Uzbekistan. Finally, this investigation is finalized with strong recommendation and suggestions.
\end{abstract}

Keywords : the use of fiber-optic sensors, methods for measuring parameters, deformation, rotation speed, fiber-optic communication lines (FOCL), reflectometers (RFTS).

\section{INTRODUCTION}

According to statistics, the amount of information transmitted in the world and communication services provided is increasing exponentially, with real demand constantly exceeding the forecast. The fast and reliable transmission of data, voice and video has gained exceptional importance in recent years for stable control. Today, the main transmission medium is optical fiber, because it has one of the main advantages over other transmission media - a virtually unlimited bandwidth resource. In this regard, the problem of FOL fiber reliability comes to the fore. Its solution includes a wide range of issues, one of which is the organization of prompt and efficient servicing of optical cables, as well as the timely diagnosis of their condition [1-3].

Intensive development of telecommunication technologies and high competition between telecom operators using fiber-optic communication lines (FOCL) put forward the task of centralized control over network fiber-optic links in order to timely detect and quickly troubleshoot optical cables.

The basic idea underlying early diagnostics is that it is necessary to control the most dangerous factor for the state of an optical fiber - its mechanical tension. When the relative elongation of the fiber is $0.3 \%$, it can function reliably for 25 years or more, whereas in the case of relative elongations and

Revised Manuscript Received on October 15, 2019.

* Correspondence Author

Nushtaeva Ozoda Xasilovna, Electronics and Radio engineering Department, Tashkent university of information technologies named after Mukhammad al-Khwarizmi, Tashkent, Uzbekistan, +99890168747,

(e-mail: nuna_x@mail.ru).

Alimjonov Botirjon Abdulmaqsud o'g'li, Electronics and Radio engineering Department, Tashkent university of information technologies named after Mukhammad al-Khwarizmi, Tashkent, Uzbekistan, +998909713993.

(e-mail: Sardorjabbarov.sj@gmail.com).

Yusupova Muhtabar Muminjanovna, Electronics and Radio engineering Department, Tashkent university of information technologies named after Mukhammad al-Khwarizmi, Tashkent, Uzbekistan, +998998278860,

(e-mail:m.rustamova@mail.ru ) more than $0.6 \%$, failure can occur during the first year of operation.

The territory of Uzbekistan is one of the most seismically active regions. According to satellite measurements, anomalously high values of the crustal displacement velocity are noted. The speed of modern movements reaches 10-12 $\mathrm{mm} /$ year, and in local areas $20 \mathrm{~mm} /$ year. Therefore, it is extremely important to study the modern geodynamic activity of the earth's crust in the areas of construction of communication lines

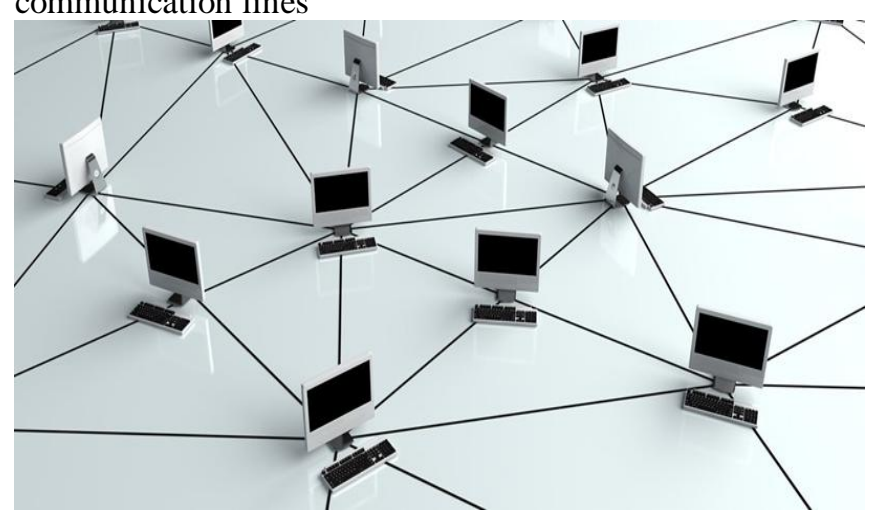

Fig.1 Network Management \& Advanced Troubleshooting

The automatic monitoring system allows you to: quickly restore cable performance by quickly detecting faults and accurately determining the location of damage; prevent failures due to the prediction of the state of the FOCL; have full line documentation; exercise centralized control with the involvement of only one specialist; significantly reduce test equipment costs.

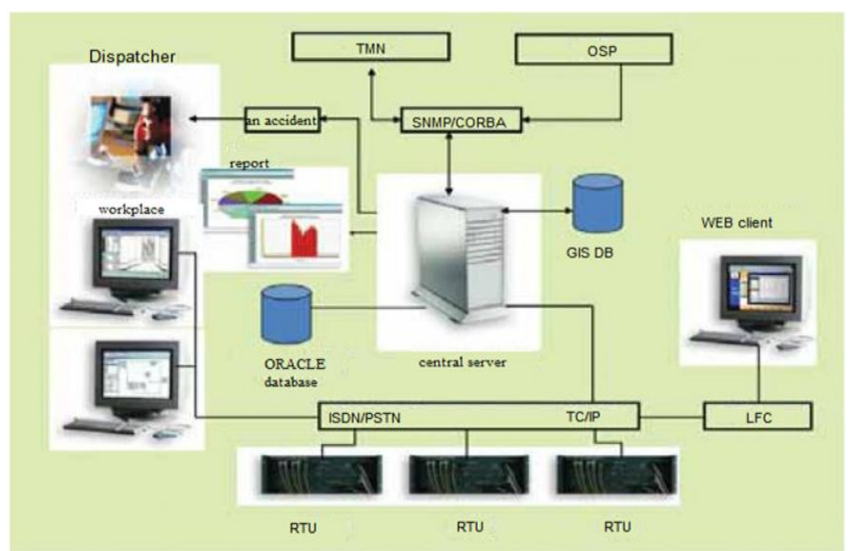

Fig.2 Atlas system architecture 
One of the most significant and practically important achievements of quantum electronics was the development of fiber-optic communication lines (FOCL) and various communication systems based on them.

Nowadays, the intensive development of fiber-optic communications, high competition between telecom operators and the cost of information resources transmitted over telecommunications networks, put forward the task of centralized control over network fiber-optic transmission lines (FOL) for the purpose of documenting and timely detection. and the speedy elimination of damage in them. Therefore, recently, telecom operators are beginning to invest considerable funds in the development of the infrastructure of their fiber-optic networks with an emphasis on the introduction and improvement of management systems and automatic monitoring of the state of communication lines and channels.

Regardless of the optical fiber control method, such systems should provide:

- remote monitoring of fiber optic cable parameters;

- reliable and timely documentation and reporting;

- automatic detection of faults in fiber-optic lines and communication channels with indication of their exact location;

- monitoring and controlling the process of notification of damage to optical cables,

- measurement of parameters of optical fibers in the automatic mode; cables.

- prediction of changes in the parameters of optical

The method of transmitting digital data streams over fiber-optic communication lines relates to communication technology and can be used both for building backbone transmission systems and for intrasite local data acquisition and control systems. The technical result consists in increasing the noise immunity and reliability of the transmitted information, reducing the requirements for the optical fiber of the fiber-optic path, increasing the speed of information transmission, increasing the protection of the transmitted information. For this information data streams are divided into blocks of fixed size, the so-called clusters, which are encoded with special optical signals in the form of successive groups of optical pulses. The number of pulses in groups can be different; within a group, each pulse is characterized by its own individual wavelength of optical radiation. Coding optical signals are combined into frames, followed by marker optical pulses with a fixed wavelength of optical radiation that is different from the wavelengths of the coding optical signals. Frames are transmitted via the fiber-optic path. At the receiving end of the fiber-optic path, reverse transformations are performed, informational data flows are restored in their original form.

\section{HELPFUL HINTS}

One of the most promising methods for measuring the tension of fibers from a practical point of view is the use of the principles of Brillouin reflectometry. The main problem for their widespread introduction is the extremely high cost of measuring equipment. Therefore, the study of the possibilities of using alternative methods and the search for new technical solutions that allow
To carry out such measurements with simpler means are highly relevant tasks. They necessitate the creation of mathematical models and their implementations in the form of computer programs, the search for technical solutions aimed at simplifying Brillouin reflectometers in order to optimize their technical and economic indicators, as well as creating simulation models that allow experiments in this area [6-8].

In the cable production process, various process deviations from the norm can occur, with the result that tensile forces are applied to the fibers. Special attention should be paid to the process of cable transportation and its installation on the FOL route. If during storage, and especially - during transportation of cable drums, they were accidentally laid on its side (that is, their axis is perpendicular to the earth's surface), which is unacceptable, then due to the uneven movement of the cable turns in the direction of this axis, considerable efforts can occur. When laying on the highway, there may be cases of dangerous effects, the risk of occurrence of which is characteristic of the process of laying the cable in the ground, in sewer pipes, pendants on supports, etc. Being in the composition of the constructed VOLP, the cable laid in the ground or in the sewage system may experience stretching due to various deformations and displacements of the ground, for example, landslides. In a suspended state, it can be subjected to tensile forces due to icing. The above list does not exhaust all possible situations that may result in the tension of optical fibers inside the cable.

Long-term observation of changes in attenuation distribution along the length FOL fibers can be operated using RFTS systems. In such systems, pulse reflectometers are used for this purpose, through which continuous monitoring of the state is performed [4-6].

Seismic monitoring of communication lines will determine the distribution tension along the fiber length and localize cable sections with dangerous values of relative elongation. Such control should be carried out at all stages of the construction and operation of fiber optic links.

With predictive maintenance, regular automatic accumulation in the database of characteristics and results of optical fiber measurements (attenuation, loss, trace, etc.) is performed, which in turn allows identifying and eliminating violations before a breakdown occurs, leading to a break in communication.

One of the most promising approaches to measuring fiber tension is to use the principles of Brillouin reflectometry. The method of Brillouin reflectometry is practically the only way to measure the absolute tension of an optical fiber. This method is most convenient in all cases when it is necessary to obtain the distribution of fiber tension along its length.

The main problem for their widespread implementation is the extremely high cost of measuring equipment.

For a simpler solution of this problem, remote optical fiber testing systems (RFTS) have been developed, the principle of operation of which is based on the use of optical reflectometers (Optical Time Domain Reflectometer (OTDR)), which measure the parameters of communication line for 
backscattering of a light wave propagating in passive or active fiber optic cable. In the first case, a backup optical fiber is tested, the result of which is judged on the health of the entire cable. In the second case, the fiber is being tested over which information is transmitted, and therefore, optical radiation with a wavelength different from the radiation wavelength used for information transmission is introduced into the communication line, and these waves are separated at the receiving side.

Such systems should provide:

- remote monitoring of fiber optic cable parameters;

- reliable and timely documentation and reporting;

- automatic detection of faults in fiber-optic lines and communication channels with indication of their exact location;

- monitoring and managing the process of notification of damage to optical cables;

- measurement of parameters of optical fibers in the automatic mode;

- prediction of changes in the parameters of optical cables.

The purpose of early diagnostics of fiber-optic lines is to identify promising methods of reflectometry, as well as to study the functional capabilities of the Brillouin reflectometry method applied to the diagnosis of fiber optic links.

- It is obvious that the use of these methods allows a new approach to the question of testing optical cables and maintenance of optical networks, providing:

- the average time of localization and identification of faults fiber optic lines not exceeding 10 minutes;

- - forecast of possible network faults, and the systematic accumulation of measurements of optical elements;

- the ability to compare the database of measurement results with the results of current measurements.

Thus, these methods provide automatic detection, accurate localization and indication on the geographic map of the existing fiber optic fault, which allows you to immediately send a repair team to carry out restoration work and minimize the cost of technical operation of fiber - optic communication lines.

At the same time, the qualification of the engineering and technical personnel must ensure the implementation of complex procedures for testing equipment, including in the field.

\section{CONCLUSION}

Summarizing the above, we note that the use of an automated FOC administration system allows for automatic detection, accurate localization and indication of the existing fiber optic fault on a geographic map, allowing you to immediately send a repair team to carry out restoration work and minimize the time to repair optical cable faults, which include

- Cable breaks due to construction, fire, road and rail accidents, natural disasters or sabotage;

- faults caused by failure of fiber optic components, poor-quality welded joints, increased losses due to moisture ingress into the cable, violation of switching in the network, etc. [3].

The introduction of an automatic monitoring system

is inextricably linked with the creation of a cable management database for a fiber-optic network. Due to its centralization, flexible scalability and configurability, this contributes to increasing the efficiency of managing complex and branched fiber optic links, simplifying the tasks of documenting new cables and changing the status of existing ones.

The components of the system have a modular design with a wide range of different configurations and sets of interfaces; therefore, the monitoring system is easily incorporated into any telecommunication network, built up and adjusted to its current changes.

With growing competition in the telecommunications industry, the problem of constantly maintaining the high quality of the services provided looms more clearly. The Orion system allows you to optimize the average recovery time of fiber optic links in case of failure and is able to identify the wok faults before they affect the quality of communication. Thus, it provides a high level of service for the fiber-optic communication network, saving investment and helping to improve the quality of service for end users.

Orion system provides efficient use of human resources. When a malfunction occurs, the system detects it and localizes it. The recovery team is sent directly to the injury site with detailed information on the accident. Since, as a result, the recovery time is significantly reduced, human resources can be allocated more efficiently and, ultimately, the cost of maintaining the health and high reliability of the communication system is reduced.

\section{REFERENCES}

1. Features of the use of optical switches in modern information networks. Gaivoronskaya G.S., Ryabtsov A.V. Ukraine 2015

2. Makarov S. B., Zavyalov S. V. Improving the noise immunity of coherent reception of non-orthogonal multi-frequency signals. // Scientific and technical statements of St. Petersburg State Polytechnic University. Computer science. Telecommunications. Control. - Issue 2 (193) / 2014

3. Vishnevsky V., Lyakhov A., Portnoy S., Shakhnovich I. Broadband wireless information transmission networks. M.: Eco-Trend, 2005.

4. B.N.Rakhimov, T.D.Radjabov. Some Aspects of Improvement of Automatic optoelectronics colorimeter Receiver of Optical Radiation (ROR) //RFBR and DST Sponsored "The Second Russian-Indian Joint Workshop on Computational Intelligence and Modern Heuristics in Automation and Robotics". -Novosibirsk, Russia, 2011.

5. B.N.Rakhimov, Z.T.Khakimov, X.G.Gaziyev, D.E.Sultonova. Calculation of technical characteristics mainline fiber optic link// Perspectives for the development of information technologies ITPA -2014 Tashkent. c. 370-375.

6. Zak E.A. Fiber-optic converters with external modulation. M. Energoatomizdat, 1987, p. 11-15.

7. BN Rakhimov, TD Rajabov. Optoelectronic system for detection of pre-destruction of objects and structures by means of optical fibers. Scientific and Technical Journal Vestnik TUIT, 2011 №2 C 35-39. Tashkent.

8. Ovcharenko AB, Kuznetsov AS, Trushin V. D. Application of the OTDR in fiber optic systems for controlling the integrity of aircraft structures from composite materials / Proceedings of the VIII Symposium on Automation Problems in a Strength Experiment, Novosibirsk, 1990. - P. 109-112.

9. BN Rakhimov, TD Rajabov. Optoelectronic monitoring system for composite building structures based on fiber-optic cables "Axborot texnologilari va telekom-munikatsiya muammolari" Republic of scientific and technical confrontation. 21-22 April 2011 in Tashkent.

10. B. N. Rakhimov, T. D. Radjabov. Republican scientific and technical conference "Information technologies and telecommunication problems", which is a composite design of the optoeloconometers. April 21-22, 2011 Tashkent. 